\title{
The Moon
}

\author{
Ross Taylor ${ }^{1}$
}

Received: 13 May 2015/Revised: 14 July 2015/Accepted: 10 November 2015/Published online: 27 November 2015

(C) The Author(s) 2015. This article is published with open access at Springerlink.com

\begin{abstract}
Recent geochemical and geophysical data from the Moon enable a revision of earlier interpretations regarding lunar origin, structure and bulk composition. Earth and Moon show many similarities among their isotopic compositions, but they have evolved in totally dissimilar ways, probably related to the deficiency of water and volatile elements in the Moon as well as the vast differences in size and internal pressure. Some global geochemical differences from the Earth such as volatile depletion based on K/U ratios have been established. However, all current lunar samples come from differentiated regions, making the establishment of a bulk composition more reliant on bulk geophysical properties or isotopic similarities; it remains unclear how the latter arose or relate to whole Moon composition. The lack of fractionation effects among the refractory and super-refractory elements indicates that the proto-lunar material seems unlikely to have been vaporized while the presence of volatile elements may place lower limits on proto-lunar temperatures. The apparent lack of geochemical evidence of an impacting body enables other possible impactors, such as comets, to be considered. Although the origin of the Moon remains currently unknown, it is generally believed that the Moon originated as the result of a giant impact on the Earth.
\end{abstract}

Keywords Lunar origin - Grand tack model - Apollo . Volatite elements

The commentary to this article is available at the doi:10.1007/s11631015-0083-1.

\section{Ross Taylor}

ross.taylor@anu.edu.au

1 Australian National University, Canberra, Australia

\section{Introduction}

Despite decades of research following the samples returned by the Apollo Missions, the Moon remains an enigma with currently little consensus on lunar composition or on the origin of our satellite. For the past 25 years, scientists have accepted that the Moon formed as a result of the low-angle collision between the Earth and another body about the size of Mars. Such a scenario solved many problems but predicted that the Moon should contain a significant amount of material from the impactor. It has been generally held, based mostly on meteorite evidence that possible Moonforming impactors would likely possess isotopic ratios (notably in oxygen) that differ from those of Earth. Models have usually shown that the impacting body contributes most of the material now in the Moon. Thus it has been surprising that recent isotopic analysis of lunar samples has revealed little trace of material other than that resembling the Earth.

\subsection{Pre-apollo ideas}

The Moon is in plain sight, accessible to naked-eye observation by keen-eyed observers. This has led to its use an early calendar and to many religious ideologies. The dark areas that make the features of "The Man in the Moon" are obvious while binoculars or a low power telescope show the craters. Galileo in 1609 saw that the Moon was mountainous like the Earth. William Herschel, the 18th Century discoverer of Uranus, thought that the Moon was inhabited. Some workers before the Apollo missions continued to believe in the existence of microbial life beneath the surface.

When the lunar orbit, mass and density were established, it was clear that the Moon was less dense compared to the 
Earth. The low density of the Moon indicated that it was probably depleted in metallic iron compared to the Earth. Its orbit was not equatorial but inclined at $5.1^{\circ}$ to the plane of the ecliptic and the Moon did not have any atmosphere.

Two areas were immediately obvious to casual observation. The most extensive was white and covered with large craters. The other surface feature that often occurred in semi-circular patches was smooth and dark, with many fewer and smaller craters. It was also solid. Some thought that these plains were composed of lava, although the apparent absence of terrestrial-like volcanoes was strange.

The Moon was large for a satellite relative to its planet compared to other the moons of comparable size that occur around the giant planets. Venus, our "twin planet", lacked a satellite.

Thus the Moon was very puzzling to terrestrial observers.

There was a general belief before Apollo was that we could discover much about the origin of the solar system by going to the Moon. This was a major scientific justification for the manned lunar missions, although they were mainly driven by political considerations. Ranger, Orbiter and Surveyor missions, designed to provide information about the nature of the lunar surface, preceded the Apollo 11 landing in 1969. Nothing had been settled after 300 years of speculation but this changed with the arrival on Earth of a few samples from the Apollo Missions, that in turn provided us with new puzzles.

\subsection{The Apollo Missions}

The Apollo Missions showed that the surface of the Moon is covered with a blanket (or regolith) a few meters thick of rubble and dust formed from the impacts of meteorites, resulting in a rounded rolling surface. The absence of familiar landmarks made it extraordinarily difficult to judge distances. There is a surprising amount of relief, over $16 \mathrm{~km}$ between the highest and lowest point (Smith et al. 2010). This rugged terrain is the consequence of giant impacts, rather than due to the forces of plate tectonics that shape the surface of the Earth Plate tectonics is absent on the Moon.

The Moon has a crust, several percent of planetary volume that formed, shortly after the formation of the Moon that occurred about 100 million years after the formation of the solar system. The crust, only about $30-40 \mathrm{~km}$ thick as established by the GRAIL mission, (Smith et al. 2010) varies widely in thickness, reaching $100 \mathrm{~km}$ on the far-side, on a body whose radius is only $1738 \mathrm{~km}$.

The lunar highland crust is different in composition to that of the interior and contains a large proportion of calcium feldspar which is responsible for the white color of the lunar highlands. It is complex in detail, mainly because the rocks were smashed up by meteoritic bombardment. However, their overall chemical composition seems mostly to have survived the bombardment. The studies of the returned lunar samples and of the lunar landscape demonstrated the importance of large impacts of asteroids, meteorites and comets in the early stages of the formation of the solar system and revealed that the terrestrial planets had formed from the coalescence of large bodies rather than from fine dust.

One of the most striking features of the lunar surface is the evidence of meteorite impacts at all scales, from large basins, ranging from hundreds to over a thousand kilometers in diameter, often with concentric rings of mountains, down to tiny micron-sized pits caused by micrometeorites hitting grains lying on the surface.

Although the larger craters were long thought to result from volcanic activity, their origin due to the impact of asteroids, comets and meteorites was established not long before the Apollo Missions, after a long controversy over whether the rugged face of the Moon had been shaped by internal volcanism or by the external impacts of meteorites, asteroids and comets.

\subsection{Lunar cataclysm?}

After the volcanic versus impact debate was settled in favor of the latter, a new controversy soon arose. Dating of the major lunar basins, such as Imbrium revealed that their apparent ages clustered around 4 billion years ago, about 500 million years after the Moon formed. This led to the concept that there had been a cataclysm or spike in the impact rate, involving many large basin-forming impactors striking the Moon around that time (Taylor 1975, 1982; Norman 2009). Other ideas were that the giant basins represented the terminal stages of the accretion of the Moon and represented the final sweep-up of planetesimals that had formed both the Earth and the Moon. But the absence of older pre-4 billion ages among glasses formed during impacts supported the idea of a cataclysm or spike. This left unanswered the question of where the impacting bodies might have been stored for 500 million years and whether or not there was a cataclysm or spike in the cratering record remains currently unresolved Figs. 1, 2, 3 and 4.

One possible solution that could produce a spike in the cratering record is another model that calls for movements among the giant planets, referred to as the Nice Model (after the city in France where the concept arose) or the Grand Tack Model (Taylor 1012) so we need a brief excursion into solar system dynamics at this stage as everything is related to everything else in the solar system. 
Fig. 1 Snow line

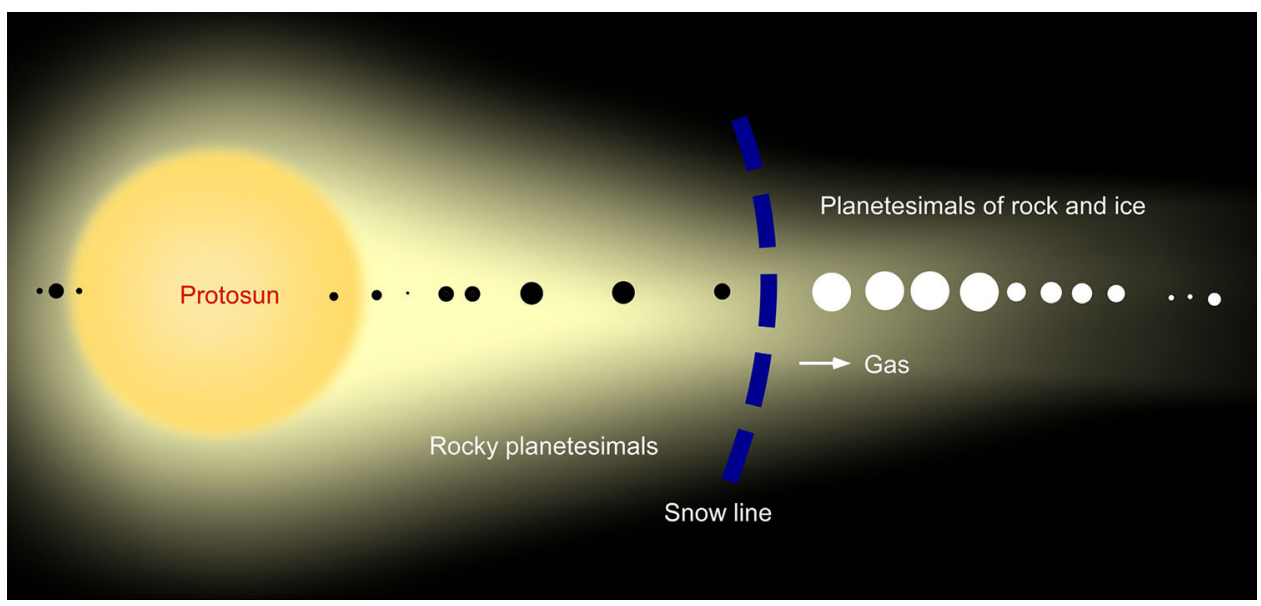

Fig. 2 One stage in the grand tack or nice model

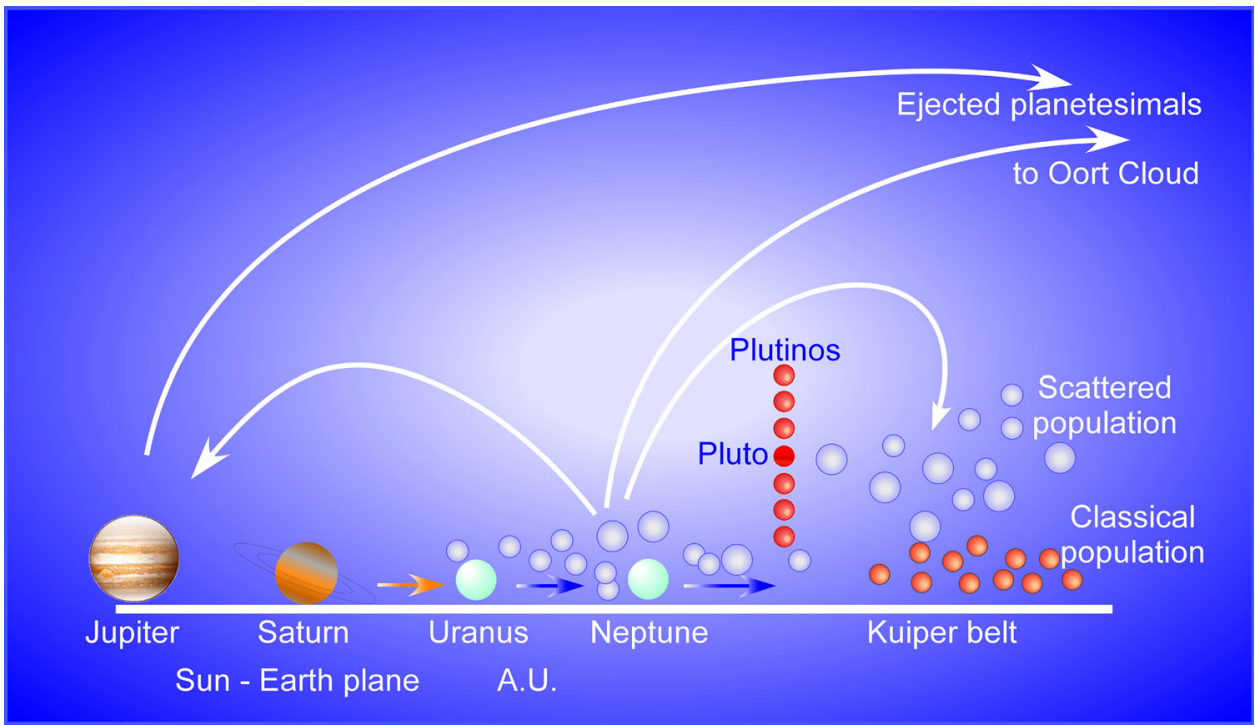

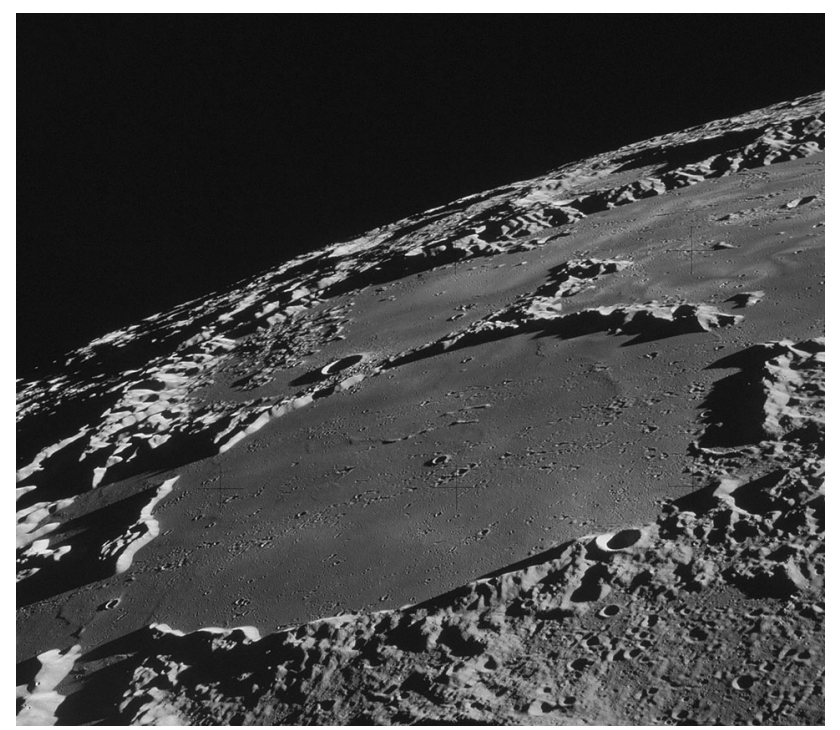

Fig. 3 Mare Ingenii

\subsection{Grand tack model}

The model is briefly described here as it might account for several of the observed features of the solar system, particularly in the outer reaches (Morbidelli and Levison 2003) According to this model, Jupiter formed first in the solar nebula at about 3.5 AU. The cores of Saturn, Neptune and Uranus, formed in that order near the snow line (the point in the primitive solar nebula at which the temperature is low enough so that water ice condenses).

As the early Sun turned on its nuclear furnace, strong stellar winds swept water and other volatile material out to between three and five AU, where the water condensed as ice and piled up in a snow line. This increased the density of the nebula at this location and so enabled icy cores about ten times the mass of the Earth to grow quickly. Two of these large cores then captured some of the gas that was also being driven away from the violent early Sun and 


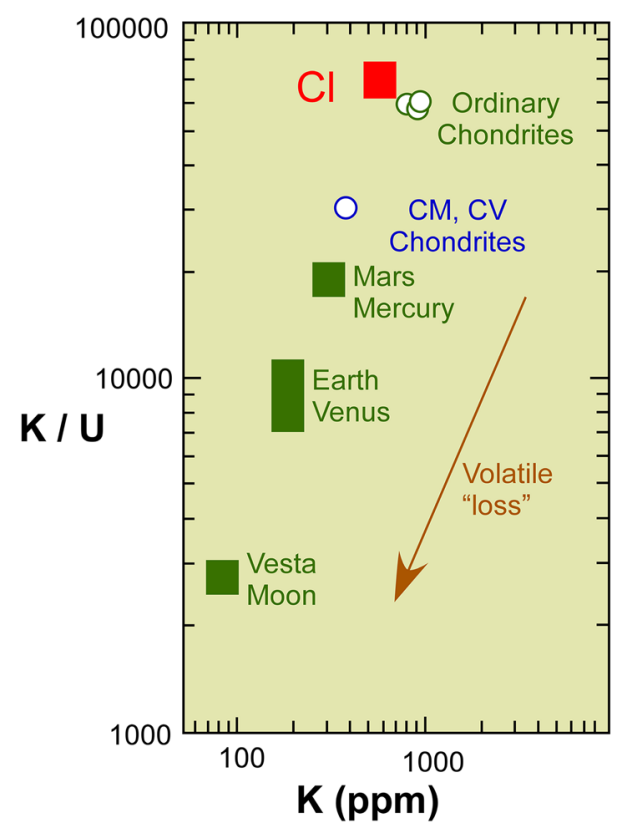

Fig. 4 Volatile element depletion in the inner solar system

became the gas giants, Jupiter and Saturn. The cores of Uranus and Neptune, farther out, only managed to catch a little gas. The terrestrial planets formed from the dry rock rubble left sunwards of the snow line (Adapted from a slide, courtesy John Wood)

Beyond Uranus lay the remnants of the nebula, a cloud of thousands of icy planetesimals (about 35 Earth-masses) that extended out to about $35 \mathrm{AU}$, a little past the present orbit of Neptune.

Encounters of these planetesimals with the giant outer planet, Uranus scattered the orbits of these small icy bodies. Originally they were scattered inwards and the planets moved out a little, to conserve angular momentum. As the small icy bodies moved inwards, so successively encountering Neptune and Saturn, these giants in turn shifted outwards. These gravitational changes were minute but slowly accumulated.

Finally, the planetesimals interacted with Jupiter. The mighty gravitational field of Jupiter scattered these tiny interlopers far and wide, some into elliptical orbits and others out of the solar system.

These slow movements might have continued indefinitely but after several hundred million years into the model, Jupiter and Saturn moved into a 1:2 resonance. Their orbits become eccentric, creating havoc and destabilizing the whole system. Jupiter wielded its great gravitational influence, pushing Saturn out to its current location. Saturn in turn swept Neptune and Uranus into eccentric orbits. These ice giants drove out into the great disk of icy bodies, a process that destroyed much of the original solar nebula. Neptune and Uranus swapped orbits in the mayhem, but finally their orbits damped down into their currently near circular form through interacting with the residual planetesimals.

A representation, not to scale, of one stage of the migration of the giant planets according to the Nice Model. Saturn, Uranus and Neptune are moving outwards, scattering the residual icy planetesimals out into the Kuiper Belt and the Oort Cloud. Some, including Pluto and the Plutinos have been captured into resonant orbits with Neptune. (adapted from Morbidelli, A. and Levison, H., Nature, Vol. 422, p. 31, 2003).

As Neptune moved out by perhaps $10 \mathrm{AU}$, it scattered the remaining icy bodies. Some, Pluto being the famous example, were moved into resonant $(2: 1)$ orbits with Neptune, while the icy giant captured Triton, a near cousin of Pluto, as a satellite of Neptune. Other icy bodies were sent into highly inclined and eccentric orbits, forming the scattered population of the Kuiper Belt. This sent a shower of impactors sunwards that resulted in the Late Heavy Bombardment (LHB) or Lunar Cataclysm, whose effects are so dramatically evident on the Moon. But this model is crucially dependant on timing of the movements of the giant planets and whether this model can account for the possible Late Heavy Bombardment on the Moon at about 4000 million years ago remains to be demonstrated.

\section{Subsequent events}

Meteorite impacts have occurred at a much slower rate on the Moon since the termination of the Late Heavy Bombardment. The youngest such major event on the Moon was the formation, about 100 million years ago, of the crater Tycho, $85 \mathrm{~km}$ in diameter. Tycho (Wilhelms 1987) formed due to the impact of a small mountain-sized body, a few kilometers in diameter. Material ejected during this impact forms the bright rays, which extend across the visible face, and form such a spectacular feature of the full Moon. The Moon contained many other surprises for geologists and geochemists. These taught us that each body in the solar system has a distinctive history.

\subsection{Geochemical evolution of the Moon}

The broad features both of lunar evolution are well understood and known better than for the Earth. Most of the Moon, was melted shortly after it formed (Taylor 1973; Pritchard and Stevenson 2000). This vast mass of molten rock has been termed the "magma ocean" and a highly energetic and rapid mode of origin, such as provided by the Giant Impact model, seems required to produce it. The best evidences for the magma ocean are the complementary relationship between the distribution of the rare earth 
element, europium, that is enriched in the highlands and depleted in source region of the mare basalts, and the great enrichment of incompatible elements in the KREEP component, that have to be derived, from mass balance considerations, from most of the Moon.

The cooling and crystallization of this ocean of melted rock in the Moon is understood in principle. Early-formed minerals such as Mg-rich olivine and orthopyroxene crystallized and sank. Feldspar, forming a little later, floated, forming a crust, due to the low density of the feldspar crystals and the dry nature of the silicate melt. "Rockbergs" of feldspar may have swept together like icebergs by tidal effects or convection currents. This might account for the differences in crustal thickness between the near and far sides. Other plausible models suggest a pile-up of debris from the early collision that formed the gigantic South PoleAitken Basin, that is $2500 \mathrm{~km}$ in diameter and $13 \mathrm{~km}$ deep.

The lunar rocky mantle was fully crystallized within a few million years. Zones formed of different minerals, from which the basaltic lavas that darken the face of the Moon were derived much later. The final dregs resulting from the crystallization of the Moon were highly enriched in all those incompatible elements that could not fit into the common minerals. This material, referred to as KREEP from potassium $(\mathrm{K})$, rare earth elements (REE) and phosphorus (P), was mixed into the crust by impacts and accounts for the very high concentrations of elements such as potassium and uranium in the crust of the Moon (Taylor 1975, 1982). However, in the absence of the recycling that accompanies plate tectonics, this material was neither subducted nor concentrated into ore deposits.

In this view, the Moon has a simple history with the mafic material now in the highlands resulting from mantle material probably excavated from beneath the thin crust during large basin-forming collisions (Melosh et al. 2014)

\subsection{The lunar highlands}

These constitute the original crust of the Moon formed shortly after its formation about 100 my after the formation of the solar system. They are the type example of a Primary Crust (Taylor and McLennan 2009) formed at or soon after accretion. Controversy still continues over whether it was formed by flotation of feldspar in a dry magma ocean or whether it was emplaced by 'serial magmatism' an analogy suggested by the terrestrial continental crust. Pure anorthosite is widely distributed as a component of the lunar crust over much of the Moon (Nagaoka et al. 2014)

The feldspathic crust was emplaced shortly after accretion and intruded by late liquids (KREEP named from its principal components of $\mathrm{K}, \mathrm{REE}$ and $\mathrm{P}$ but containing many other incompatible elements, the residual dregs from the crystallization of the magma ocean).
While the feldspathic crust and cumulate source regions for mare basalts seem to be globally distributed (Wieczorek 2013; Giguere et al. 2000), the late-stage residual melts sampled as KREEP seem not to have been distributed symmetrically but instead were concentrated around the western limb of the near side of the Moon in the vicinity of the Procellarum basin (Jolliff et al. 2000; Gross et al. 2014). KREEP seems to have been mostly restricted to the near side so that three distinct provinces have been recognized, such as the Procellarum KREEP Terrain (PKT), the Feldspathic Highlands Terrain ((FHT) and the SouthPole-Aitken Terrain (SPAT) (Jolliff et al. 2000), the last two terranes being poor in the KREEP component.

Although the average composition of the feldspathic crust remains somewhat uncertain, it clearly requires a large-scale fractionation that is more consistent with a lunar magma ocean than, for example, construction of the crust by serial intrusion and local differentiation of discrete batches of magmas produced by smaller degrees of melting of a primitive lunar mantle (e.g., Walker 1983; Solomon and Longhi 1977), which might produce compositional differences among elements such as the REE. These elements (REE, apart from Eu) are surprisingly uniform in the lunar highlands crust. The feldspathic crust was also shattered by large asteroid collisions that may have been concentrated around 4.1-3.8 b.y. (Tera et al. 1974; Gomes et al. 2005, see Norman 2009 for a recent review) but may have begun somewhat earlier (Norman and Nemchin 2014). The Highland crust contains a mafic component, usually ascribed to subsequent intrusions (e.g., Shearer et al. 2015). How much this bombardment added to or affected the petrologic complexity of the crust (possibly via differentiated melt sheets) remains an open question.

But the crust is thin and much of the mafic component might be derived from the mantle by basin-forming impacts. Melosh et al. (2014) and Miljkovic et al. (2015) have pointed out that the mafic component in the lunar crust might be derived as ejecta from beneath the thin crust. The sparse occurrence of olivine in reflectance spectra has usually been interpreted to indicate the there is little mantle ejecta in the crust. However, this apparent lack of mantle material could be explained if orthopyroxene rather than olivine is a major component in the upper mantle (Khan et al. 2006). This could be consistent with a simple lunar evolution where a crust of pure anorthosite floated and was contaminated by mafic material from the mantle excavated by large impacts into a thin crust.

\subsection{The Mare}

These dark regions (the maria) form the familiar dark features of the "Man in the Moon". The maria, although prominent visually, form only a thin veneer on the thick 
highland crust and constitute less than about $1 \%$ of crustal volume. They form the type example of a Secondary Crust (Taylor and McLennan 2009) formed by melting in the planetary mantle and eruption of lavas, long after accretion of the body.

The mare are composed of basalt and over 25 distinct varieties have been sampled, pointing to derivation from a very heterogeneous mantle.

Lavas rise to the surface of the Moon because the liquids are less dense than the surrounding solid rock. Lavas on the Moon are more common on the near side where they can more easily reach the surface because the crust is thinner. In contrast, they are rare on the far side of the Moon because they mostly fail to reach the surface through the much thicker crust. The lavas fill various impact basins and craters to differing levels.

The basalts come from many distinct regions in the mantle of the Moon, in contrast to the common basalts (MORB) erupted on Earth at the Mid-Ocean Ridges. These terrestrial lavas have a much more uniform compositions than their lunar counterparts. The lunar lavas that were sampled are also very ancient, mostly erupted between 3800 and 3200 million years ago, in contrast with the much younger age of our terrestrial Mid-Ocean Ridge Basalts.

The lack of our familiar volcanoes on the lunar surface is a result of the very fluid nature of the iron-rich lunar lavas (Taylor 1975, 1982). This enables them to flow as easily as oil for hundreds of kilometers on slopes of only a degree or two, making the remarkably flat lunar plains. This was unexpected. Basalt lavas on the Earth are viscous, flowing more like toffee than engine oil.

The basaltic lavas were erupted from deep within the Moon. They formed by the melting of zones of differing composition. These zones formed during the solidification of the Moon, as various minerals precipitated as the Moon cooled. Some contained radioactive elements that slowly heated the surrounding cumulate zones, eventually melting the more easily melted minerals, forming a liquid slush or magma. Because this basaltic melt was of lower density than the mantle, it rose to the surface and flowed out as lava. Some melts had entrained traces of volatile elements that sprayed out as fire fountains, producing many tiny globules that astronauts found as the famous green and orange glasses lavas (Taylor 1975, 1982).

The ages of these eruptions were dated as occurring between 3.9 and 3.1 billion years ago but subsequently evidence has been found of basaltic fragments 4.2 b.y. old in breccias while mapping has revealed apparently younger flows, perhaps some being only 100 m.y. in age (Braden et al. 2014)

The mare basalts are derived from a mineralogically heterogeneous interior (Taylor and Jakes 1975) and their compositions are so diverse that none could be considered as representative of the bulk lunar interior. The relative masses and distributions of their respective source regions are so poorly constrained that they provide little direct information about bulk lunar composition. Mare basalts have FeO contents $(\sim 16-25 \mathrm{wt} \%)$ that are high relative to terrestrial basalts, and are more analogous to basalts from Vesta and Mars. On this basis, the FeO content of the lunar mantle is often thought to be $13 \mathrm{wt} \%$ compared to $8 \mathrm{wt} \%$ for the Earth (e.g., Hood and Jones 1987) but if the lunar basalts are derived from FeO-rich cumulates, then they are unlikely to be representative of the bulk lunar mantle.

The deep interior of the Moon is presumably rich in $\mathrm{Mg}$ rich olivine or orthopyroxene, the first minerals to crystallize from the magma ocean, but these deep cumulate zones may be inaccessible to direct sampling unless mantle overturning occurred. In addition, both the depth of primary melting of the lunar mantle associated with the magma ocean and the presence of any remaining primitive mantle are poorly constrained.

\section{Core}

There is little evidence about the deep lunar interior (Khan et al. 2014; Raevskiy et al. 2015) although a partially molten zone may surround a metallic core. There is good evidence for the existence of a core (Belashchenko and Kuskov 2015) but it forms only a few percent of the volume of the Moon. Metallic iron and its associated elements, (nickel, cobalt, platinum, iridium etc.) are between 20 and 50 times lower than in the Earth's mantle and are possibly mostly in the lunar core (e.g., Day et al. 2007, 2010; Rai et al. 2014). Among the other surprising results from the Apollo samples was the demonstration of ancient magnetic fields, now extinct. The most likely possibility is that the field was generated internally during the freezing of the tiny fluid iron core (Weiss and Tikoo 2014).

We now understand what we are looking at on the surface of the Moon. The contrast between the lunar highlands and the lunar maria is well shown in this view of Mare Ingenii on the lunar farside. The rugged white regions are the primary crust of the Moon, mostly made of calcium feldspar. Large circular craters have been punched into this crust by impacts. Millions of years later these holes have been flooded with basaltic lavas erupted from deep within the mantle, producing the smooth grey plains of the maria. These have a few small craters on their surface from later impacts. The large circular crater, filled with mare basalt, is Thomson, $112 \mathrm{~km}$ in diameter, in the northeast sector of Mare Ingenii, which is $370 \mathrm{~km}$ in diameter. The sequence of events that produced this scene from oldest to youngest, is (1) formation of white feldspar-rich highland crust (2) excavation of Ingenii basin (3) formation of Thomson 
crater (4) flooding of Ingenii basin and Thomson crater with basaltic lava and (5) production of small impact craters on the smooth mare surface, including a chain of secondary craters across Thomson. (NASA AS15-8711724).

\subsection{Composition of the Moon}

The first Apollo sample return from the smooth basaltic plains of Mare Tranquillitatis found some unusual chemistry in contrast to our familiar terrestrial rocks. The Moon is strongly depleted in the most volatile elements, such as lead and chlorine, as well as the moderately volatile elements such as potassium and sodium. This volatile depletion is well shown by the very low K/U ratio of the Moon, 2500, compared with 10,000 for the Earth or 60,000 for the original nebula as shown by the CI meteorites (Taylor et al. 2006).

The widespread depletion of volatile elements in the inner solar system, displayed here as the abundance of potassium (K), a 'volatile' element relative to uranium (U) a 'refractory' element. CI gives the composition of the 'rocky' fraction of the Sun and of the primitive solar nebula. Mercury and Mars are less depleted than the Earth. The chondrites are various classes of stony meteorites. (courtesy Scott McLennan).

Thus the Moon is more strongly depleted in volatile elements than the Earth, but not compared to the asteroid 4 Vesta and is dry except for trivial amounts of water (see below).

The trace metallic elements, such as nickel and platinum, are depleted in the Moon to a similar extent as on Vesta. These elements concentrate in metallic cores.

The asteroid 4Vesta produced basalts with similar trace element patterns to lunar basalts, including strong depletions in volatile elements, within a few million years of the formation of the solar system. Thus at least one asteroid lost volatile and siderophile (metallic) elements similarly to the experience of the Moon.

Data from isotopes also tells us that both the Moon and the Earth lost volatile rubidium relative to refractory strontium very early on, much like the meteorites (eucrites) from the asteroid Vesta. This loss occurred at a very early stage in nebular history, close to the oldest dated material, usually referred to as $\mathrm{T}_{\text {zero }}$ (4567 million years ago, the date of the oldest objects (CAI, Mason and Taylor 1982) in the solar system and so used to date the origin of the system).

Potassium also has not undergone any isotopic fractionation so that the potassium now in the Moon, like that in all other measured samples in the inner solar system material, including the Earth, has not undergone significant evaporation or condensation during formation of the Moon. (Humayun and Clayton 1995).
But as the samples currently available from the Moon come from regions that have undergone substantial differentiation, the prospect of obtaining bulk lunar compositions becomes more difficult as illustrated by the following comment.

Using an Apollo set of geophysical data on crustal thickness and heat flow, Taylor (1982) concluded that the Moon had a crust about $52 \mathrm{~km}$ thick and therefore a probably higher alumina content (6 vs $3.6 \mathrm{wt} \%$ ) and $\mathrm{U}$ abundance ( $30 \mathrm{ppm}$ vs. $18 \mathrm{ppm}$ ) than the Earth (see also Taylor et al. 2006). But the data from the GRAIL Mission (Wieczorek 2013) showed that this interpretation was erroneous and that the Moon likely has an aluminous crust only $30-40 \mathrm{~km}$ thick, and therefore the Moon has a refractory lithophile element (RLE) composition broadly similar to that of the Earth (Taylor 2014). However, several open questions remain, including: the distribution of $\mathrm{Al}_{2} \mathrm{O}_{3}$ within the lunar crust and mantle, the global distribution of heat-producing elements, and whether the lunar highland crust becomes more mafic with depth, as in the case of the continental crust on the Earth (see Taylor and Wieczorek 2014) or whether the mafic material in the highland crust was derived from the mantle via basin-forming collisions into a thin crust. An additional problem is whether the crust was melted by later major impacts, producing melt sheets with possibly younger ages.

Can the bulk composition of the Moon be obtained by geochemical procedures based on mass balance of endmember reservoirs? The Moon is a highly differentiated body that melted when or soon after it formed. Early melting and large-scale differentiation of the Moon are indicated by the old differentiation ages of the lunar crust and mantle (about 4460 million years) but the timing and duration of the origin of the crust may be more complex, see Borg et al. 2011; Demidova et al. 2014; Gaffney and Borg 2014). Complementary trace element patterns in the lunar crust, mantle and KREEP reflect the relative compatibilities of these elements into the major minerals of the mantle (olivine, pyroxene, ilmenite) and crust (plagioclase), but the extreme compositions of lunar mare basalts (up to $14 \mathrm{wt} \% \mathrm{TiO}_{2}$ ), the presence of large concentrations of incompatible elements ( $\mathrm{Rb}, \mathrm{Ba}, \mathrm{REE}, \mathrm{Zr}, \mathrm{Hf})$ in the nearside Procellarum-KREEP Terrane, and the uncertain relative volumes of these highly differentiated reservoirs complicates attempts to reconstruct the lunar bulk composition by mass balance. We should recall that the thin crust of the Moon renders it possible that the mafic components in the highland crust may be derived as ejecta from the mantle during basin-forming impacts (Melosh et al. 2014; Miljkovic et al. 2015) thus adding further complexity to an already difficult problem.

Therefore estimates of the composition of the lunar interior have the status of inspired guesses. Clearly bulk 
geophysical properties, such as density (e.g., Darwin 1879) and isotopic similarities (although it remains unclear how the latter relate to bulk composition) may provide a surer guide to the bulk composition of the Moon, which appears to be broadly similar to that of the Earth's mantle, although much more depleted in volatile elements.

It is relevant here to ask here how well we know the composition of the Earth. The terrestrial mantle is often considered to be isotopically homogeneous. But it should be asked how and when did it achieve that status. In current models, the Earth is assembled from a suite of differentiated planetesimals with probable differing isotopic ratios that seem to have become homogenized later. We should recall that current estimates (Sun 1982; Taylor 1982; Taylor and McLennan 2009) on the composition of the Earth are based on lavas and xenoliths derived from the upper few hundred kilometers.

\subsection{Lack of fractionation among refractory lithophile elements}

It has generally been assumed by geochemists that the refractory lithophile elements (RLE) are not fractionated in the Earth relative to their abundances in chondritic meteorites (e.g., Palme and Jones 2005). Although recent highprecision determinations of some stable and short-lived isotopes have questioned that view in detail (see review by Campbell and O'Neill 2012), the similarities in composition (typically within a few per mil) between the Earth and various classes of chondritic meteorites combined with independent estimates of the Earth's primitive mantle based on measured samples (e.g., Sun 1982) confirms the general applicability of the 'chondritic' model for RLE in the Earth.

Lodders (2003) gives condensation temperatures for the rare earth elements showing that $\mathrm{Eu}$ and $\mathrm{Yb}$ (and to a lesser extent, Ce) are more volatile than the other REE. Taylor $(1987,1990)$ drew attention to the fact that the super-refractory elements $\mathrm{Zr}$, Hf, $\mathrm{Y}$ and the refractory elements such as $\mathrm{Al}, \mathrm{Ca}, \mathrm{Ti}, \mathrm{REE}, \mathrm{U}, \mathrm{Th}, \mathrm{Ba}$ and $\mathrm{Sr}$ with condensation temperatures above $1200 \mathrm{~K}$ are present on the Moon in roughly chondritic proportions. But they show fractionated behavior, based on volatility differences, in the refractory inclusions in meteorites (CAI, e.g., Mason and Taylor 1982). The super-refractory elements Zr, Hf, Sc, and $\mathrm{Y}$ are not fractionated in the Moon relative to the other refractory elements in contrast to their behavior in CAIs and in hibonite where they are separated from the more volatile REE. The abundance of the RLE in the Moon is generally assumed to be parallel to chondrites. Although depletions and enrichments of Eu have long been ascribed to crystal-melt partitioning, decades of study of the REE in particular show no sign of $\mathrm{Yb}$ or other anomalies that would have been expected if the material now in the Moon had been exposed to high temperatures in a vaporized disk and Taylor (1990) concluded that this placed upper limits on the temperatures to which proto-lunar material had been exposed (See also Petaev et al. 2014; Pahlevan 2013). The depletion of the volatile elements in the Moon relative to the Earth might also place some lower limits but these are difficult to quantify.

\subsection{Water on the Moon}

Water in the inner solar system seems to have arrived there by chance (Morbidelli et al. 2012). Water is essential to life as we know it, so there is an understandable interest in the presence of water elsewhere, even on a body as dry as the Moon. The NASA LCROSS Mission (Heldmann et al. 2015) successfully detected water, following the impact of the upper stage of the launch rocket into one of the shadowed craters near the south lunar pole. These traces of water, trapped as ice in the lunar soils, were delivered from comets, or from the interaction of hydrogen from the solar wind hydrogen with surface minerals.

This evidence for traces of water on the surface needs to be distinguished from reports of 'water' (present as the $\mathrm{OH}$ ion) in minerals derived from the interior of the Moon. The lunar rocks are dry and are strongly reduced. No micas or amphiboles that contain $\mathrm{OH}$, have ever been found. Ferric iron that would indicate oxidizing conditions is absent as well.

But trace levels of very volatile elements such as fluorine and chlorine have long been reported in lunar samples. Taylor (1975, 1982 and Reed and Jovanovic 1973) As both elements are much more volatile than water, it should not have been surprising to geochemists that a minute trace of water might be incorporated within the Moon along with trace ( $\mathrm{ppb}$ ) levels of many other very volatile elements.

During the crystallization of the lunar magma ocean, such elements were concentrated in residual fluids. In this process, any water would finish up in the residual melt (along with many other incompatible elements). Minute traces of water were also trapped in mineral zones in the deep interior and are now found along with fluorine and chlorine in the $\mathrm{OH}$ sites in minerals such as apatite, a trace mineral in the basalts.

Water present in apatites from the Moon has a very variable isotope ratio $(\mathrm{D} / \mathrm{H})$, higher than that of terrestrial water, in contrast to the identical oxygen isotope ratios in both bodies. This may tell us that the trace of water on the Moon probably did not come from the Earth (Barnes et al. 2014; Boyce et al. 2010, 2014). In this important but currently confusing topic, my assessment is that water is expected to be present at a few ppm in the bulk Moon but its status as an incompatible element concentrated in 
residual melts and phases and its likely heterogeneous distribution within the lunar interior, makes it impossible to calculate a meaningful value for the bulk Moon based on direct measurements. This problem is exacerbated by the presence of $\mathrm{OH}$ in trace minerals in mare basalts, which are themselves derived from cumulate zones within the mantle. Still, it would seem unusual if the water content of the Moon were similar to that of Earth's mantle considering the strong depletions of other incompatible elements of lesser volatility (such as potassium). Although portions of the Moon seem a little damp, overall the Moon is much drier than the Earth (Sharp et al. 2010; Hauri et al. 2015; Valley et al. 2014; Robinson and Taylor 2014, ElkinsTanton et al 2002; Elkins-Tanton and Grove 2011; Hauri et al. 2015). Thus the Moon seems essentially devoid of water, unless one regards the presence of a few parts per million as significant and the Moon seems drier than the Earth by large factors. The great publicity about traces of water, either on the lunar surface or in the interior of the Moon, seems to be due to the sacred-cow status that has been given to water (see also Albarede et al. 2015).

\subsection{The origin of the Moon: early notions}

The Moon is in plain sight, but it has defied efforts to explain it for hundreds of years. So the Moon presents a particularly difficult problem. How did it form and why is it there? It is sobering to record that none of the models that had been erected by three centuries of theoretical reasoning before the Apollo Missions were able to account for it.

Following the sample return, all pre-Apollo theories for the origin of the Moon failed for various reasons. Hypotheses in which the Earth captures an already formed Moon were abandoned. It turns out to be very difficult to capture the Moon into its present orbit around the Earth. In such a model, the curious chemistry of the Moon had to form somewhere far away. Putting problems out of sight does not solve them. But we should not forget the similarity between lunar basalts and the lavas from the asteroid 4Vesta and other similarities with Vesta.

The similarity in density between the Moon and the Earth's silicate mantle fuelled speculation, dating back to George Darwin (1879), one of the sons of Charles, that the Moon had formed from the Earth's rocky mantle following core formation on the Earth.

Such "fission hypotheses" that derive the material for the Moon from the Earth's mantle, although always popular, encounter basic difficulties. The spin of the EarthMoon system, although large,is apparently insufficient by a factor of about four to allow fission to occur. The Moon is also depleted in volatile elements even relative to the Earth so that high temperatures seem to have been involved. Then there is the evidence that the Moon was molten shortly after its formation. However to add further to the problem the ratios of the oxygen, chromium, titanium isotopes and those of many other non-volatile elements turn out to be identical or very close in both bodies.

Double planet models that form the Moon and Earth in association possess the twin difficulties of failing to account for the high spin of the Earth-Moon system, and of readily accounting for the density difference.

Yet another model formed the Moon from a ring of rocky debris produced by break-up of incoming asteroids as they come within the Roche Limit. This process was supposed to result in a ring of broken-up rock debris around the Earth. Their tougher iron cores stuck together and crashed into the Earth. These last two models form the Moon as a natural process related to the formation of rocky planets and so should lead to the presence of moon-like satellites around other rocky planets.

But they fail a crucial test. The absence of a comparable satellite around our twin, Venus, seems the fatal flaw in these notions. None of these theories accounted for the unique nature of the Earth-Moon system, for the strange lunar orbit and for the high spin of the Earth-Moon system. Like ships hitting an uncharted rock, they all sank from this defect. Uncommon objects like our Moon may require a unique mode of origin.

\subsection{The origin of the Moon: current ideas}

Several key features need to be explained (Stevenson and Halliday 2014).

1. The Moon has a lower density than the Earth and is large relative to its planet for a satellite

2. The Moon has most of the angular momentum of the Earth-Moon system

3. The isotope ratios for the non-volatile elements in the Moon have nearly identical values to those on the Earth. This is the major fact that provides difficulties for the Giant Impact Model

4. 4, The Moon formed close to the Earth about 100 million years after the formation of the solar system and has moved steadily away

5. The Moon melted shortly after it formed.

6. The Moon is depleted in volatile and moderately volatile elements relative to the Earth while both bodies are depleted relative to those in the primitive solar nebula.

The Moon has an isotopic composition that is identical to the silicate mantle of the Earth within the relatively tight analytical constraints provided by modern instrumentation for oxygen (Weichert et al. 2001; Kohl et al. 2015), silicon (Armytage et al. 2012), calcium (Simon et al. 2009) magnesium (Sedaghatpour et al. 2013), iron (Moynier et al. 
2006), titanium (Zhang et al. 2012), strontium (Moynier et al. 2010a) and chromium (Lugmair and Shukolyukov 1998) but it has had a totally different geochemical development. The similarity in oxygen isotopes seems particularly significant as $\mathrm{O}^{2-}$ is a large anion and the packing of oxygen anions largely controls the abundance of other elements in silicates.

The Moon is depleted in volatile elements (Wolf and Anders 1980) relative to the Earth, which in turn is depleted relative to $\mathrm{CI}$ abundances. This is shown most clearly by $\mathrm{K} / \mathrm{U}$ ratios, 2500 for the Moon relative to 10,000-12,500 for the Earth and 60,000 for CI. Potassium isotopes, however, show no difference between Earth, Moon and other inner solar system bodies (Humayun and Clayton 1995) suggesting that a major depletion of potassium in the entire inner solar system took place close to $\mathrm{T}_{\text {zero, }}$ (4567 m.y. ago) This finding is reinforced by the primitive value of the ${ }^{87} \mathrm{Sr} /{ }^{86} \mathrm{Sr}$ ratio in the Moon (LUNI, Nyquist 1977), although the value for the Earth is not well established. Although Li isotopes appear to have been fractionated by the magma ocean, they do not seem greatly different between the Earth and the Moon (Magna et al. 2006). One of the few isotopic differences between the Moon and Earth is the enrichment of the heavy isotopes of $\mathrm{Zn}$ in the Moon, which has been ascribed to volatile loss during the formation of the Moon (Paniello et al. 2012). Future isotopic work at ppm or ppb levels may resolve some of these problems

\subsection{The giant impact model}

This was the current model for a generation developed during the 1984 meeting in Hawaii and remained as the consensus for a generation until 2012 when new data appeared that raised great difficulties for the model (e.g. Stevenson and Halliday 2014; Asphaug 2014).

The main problem was that the isotopic composition for many elements in the Moon was shown to be closely identical to that of the Earth Perhaps the most significant other property of the Earth-Moon system is that its angular momentum is high compared to that of the other planets. This problem was pointed out by Al Cameron (1925-2005), who showed that this excess angular momentum had to have arisen through the collision of the Earth with an object a little larger than Mars. The high spin rate of the Earth and Moon apparently cannot arise from a series of small impacts. However, one large impact could account for it. The required conditions in the model were for the impactor (usually called Theia) to have a mass about fifteen percent of that of the Earth (larger than Mars) and to hit at a glancing angle with a velocity of five kilometers a second. This body, as well as the Earth was assumed to have formed a metallic core and rocky mantle before the collision. Both the Earth and the impactor were melted by the collision. The metallic core of the impactor separated from the rocky mantle and fell into the Earth within a few hours. The molten mantle of the impactor formed a disk around the Earth from which the Moon formed. Temperatures in the disk reached several thousand Kelvin.

The impact event was also sufficiently energetic to vaporize much of the material that went to make up the Moon. This was often supposed to explain such unique geochemical features as the dry nature of the Moon and the extreme depletion of very volatile elements. But there is little direct evidence in the Moon for condensation from a vapor phase (see above) A final consequence of the giant impact model for lunar origin was that the event was energetic enough to melt the mantle of the Earth, but the effects on the geochemical evolution of the terrestrial mantle remain to be fully evaluated.

Unique events are difficult to accommodate in most scientific disciplines. Of course, a prime requirement for this hypothesis was that there was a supply of bodies of the right size to hit the Earth. Fortunately for the model, there is plenty of evidence from the tilts of the planets and the presence of the large lunar impact basins for the previous existence of large bodies in the inner solar system.

Thus although the details of the Moon-forming event are not predictable, massive collisions early on in the solar system seem to have been common. One just happened to have the appropriate mass and velocity and hit the Earth at the right angle to produce the Moon in this hypothesis.

When did the Moon form? Although dates as early as 30 million years after Tzero have been commonly suggested, these came from an erroneous interpretation the hafniumtungsten isotopic system.

Current models suggest ages around 4460 million years ago, consistent with dates for the crystallization of the highland crust. Thus, formation of the Moon took place around 100 million years after $\mathrm{T}_{\text {zero }}$.

\section{Problems with the giant impact model}

No current model provides a satisfactory explanation for the origin of the Moon. Thought to have been settled for a generation by the Giant Impact hypothesis (e.g., Benz et al. 1989), it is once again in a state of flux with new geochemical data and geophysical models drawing a more direct link to the Earth as the primary source of proto-lunar material, and allowing a wider range of dynamical conditions that includes both larger (Canup 2012) and smaller (Cuk and Stewart 2012) impactors than the canonical Mars-sized impactor, in order to account for the angular momentum constraint. 
A general conclusion from these isotopic observations seems to be that the Moon appears to have apparently little trace of the impacting body although models continue to show that the impactor makes a significant contribution to the putative proto-lunar disk resulting from the collision (Canup 2012). A general conclusion seems to be that the Moon appears to have very little trace of the impacting body.

The origin of the Moon has always been an improbable event and seems to have become more so. The Earth was assembled, in the planetesimal hypothesis, from a wide variety of bodies, which may have been differentiated into cores and mantles, with presumably differing oxygen isotope ratios. Was the Earth homogenized in isotopic composition before the Moon-forming event or by that event? What happened to the Moon-forming impacting body and its possible core? Was it larger or smaller than the Marssized body implied by earlier dynamical models of the giant impact? (e.g., Benz et al. 1989). Was it incorporated into the Earth and homogenized or lost? Models have always assumed that the impactor was differentiated and that the core was incorporated into the Earth in order to account for the low density of the Moon compared to the Earth. However if the Moon was derived from the terrestrial mantle after core formation, the proto-lunar material was low in iron in any event. Was there indeed a vapor cloud resulting from the putative impact from which the Moon may have formed (see above, Pahlevan and Stevenson 2007; Pahlevan et al. 2011)?

No impactor seems to be needed by the geochemistry at present but a highly energetic origin is still required. Impacts by planetesimals, differentiated or not, seem unable to account for the composition of the Moon coupled with the lack of much sign of the impacting body. The Moon bears a signature of the chemistry of the mantle of the Earth but it is also both depleted in volatile elements and water.

So do we need new models of the impactor? The composition of the Moon looks like massive distal ejecta from the terrestrial mantle. The apparent absence of definitive evidence of the impacting body naturally draws attention to the possibility of a cometary impact. Perhaps only massive impacts on the terrestrial mantle by icy comets may be capable of providing the low iron content of the Moon, the necessary energy to melt most of the Moon, deplete the volatile elements, and leave little or any trace of the impactor. But comets, as impactors in the early inner solar system are not without their problems. In current models, they seem to come from the vicinity of Jupiter at least (Morbidelli et al. 2012), as rare interlopers into the dry early inner solar system. Thus they might impact at high velocities, in contrast to current models for forming the Moon that call for low velocity impacts. Indeed, if comets were involved, then the formation of the Moon becomes an even more improbable event.

\section{The parallel with tektite formation}

Another possibility seems worth considering. The lunar composition in so far as it has been established, bears a curious parallel to the compositions of tektites, notably in its depletion of volatile elements (including water), heavy $\mathrm{Zn}$ isotopic composition, and lack of a geochemical signature of the impactor. Is this a mere coincidence or does it tell us something significant about the origin of the Moon? Tektites are $\mathrm{mm}$ - to $\mathrm{cm}$-sized glassy objects produced by the impact of asteroids under some specific conditions onto the crust of the Earth (e.g., Koeberl 1986). Their RLE, notably REE, compositions broadly reflect those of nearsurface, terrestrial materials which in turn image the composition of the Earth's crust. However, like the Moon, tektites are also depleted in volatile elements, including water, (20-200 pm; Koeberl and Beran 1988) and they show debatable or barely detectable traces of the impactor. Tektites also have heavy $\mathrm{Zn}$ isotopic compositions (Moynier et al. 2009), although the isotopically heavy $\mathrm{Cu}$ in tektites contrasts with the lunar composition (Moynier et al. 2006, 2010b) and may require a different set of conditions for the volatile loss. Taylor and Koeberl (2013) drew attention to the parallels between the composition of tektites derived from terrestrial crustal rocks by relatively small meteorite impacts and that of the Moon, presumably formed by an immensely larger event involving the terrestrial mantle. These interesting comparisons imply that the Moon was derived from distal ejecta from a massive impact on the Earth but on a scale many orders of magnitude larger scale than was involved in the production of tektites. The analogy may be worth pursuing as it results in volatile-depleted ejecta from the Earth without leaving much trace of the impactor, The implication of this conceptual model is that the Moon reflects the composition of the target (i.e. proto-Earth) rather than that of the impactor as implied by earlier dynamical models and that the impacting body leaves little trace of itself.

\section{Conclusions}

Although the Earth and Moon show many similarities among their isotopic compositions, they have evolved in totally dissimilar ways, probably related to the deficiency of water and volatile elements in the Moon. Samples from the Moon are derived from highly differentiated zones, making estimates of bulk composition more reliant on bulk geophysical measurements or isotopic similarities. In view 
of the lack of fractionation effects among the refractory and super-refractory elements, the proto-lunar material seems unlikely to have been vaporized. (i.e. no condensation seems to have taken place at temperatures much in excess of the melting points of silicates) Is this a real constraint or does it merely illustrate the difficulties of modeling huge events? The formation of tektites points to another possible lunar-forming model that excavates, in the lunar case, over one percent of the terrestrial mantle (low in metallic iron), depletes the proto-lunar material in volatiles, melts much of the material and leaves apparently little or no trace of itself. Another alternative could be the impact of an icy comet, raising vast new problems for modelers.

The amount of 'pure anorthosite' revealed as well as the thinness of the crust may suggest that lunar evolution could have been a comparatively simple affair. Perhaps a simple crust of anorthosite formed from the magma ocean with the mafic component coming from the mantle by excavation in basin-forming collisions. Later impacts may remelt parts of the crust accounting for the younger ages in the highland rocks as well as mixing in of the KREEP dregs from the magma oeean.

In summary, the origin of the Moon seems to require massive impacts on the Earth as both models and geochemical studies have long suggested.

Open Access This article is distributed under the terms of the Creative Commons Attribution 4.0 International License (http://crea tivecommons.org/licenses/by/4.0/), which permits unrestricted use, distribution, and reproduction in any medium, provided you give appropriate credit to the original author(s) and the source, provide a link to the Creative Commons license, and indicate if changes were made.

\section{References}

Albarede F et al (2015) An intrinsic volatility scale relevant to the Earth and Moon and the status of water on the Moon. MAPS 50:568-577

Armytage RMG et al (2012) Silicon isotopes in lunar rocks. GCA 77:504-514

Asphaug E (2014) Impact origin of the Moon. Ann Rev Earth Planet Sci 42:551-578

Barnes JJ et al (2014) The origin of water in the primitive Moon as revealed by the lunar highlands samples. EPSL 390:244-252

Belashchenko DK, Kuskov O (2015) Molecular-dynamic modeling of thermodynamic properties of the lunar Fe-S core. Doklady Earth Sci 460:37-40

Benz W, Caneron AGW, Melosh HJ (1989) The origin of the Moon and the single impact hypothesis. Icarus 81:113-131

Borg LE, Connelly JN, Boyet M, Carlson RW (2011) Chronological evidence that the Moon is either young or did not have a global magma ocean. Nature 477:70-72

Boyce JW et al (2010) Lunar apatite with terrestrial volatile abundances. Nature 466:466-469

Boyce JW et al (2014) The lunar apatite paradox. Science 344:400-402

Braden SE et al (2014) Evidence for basaltic volcanism on the Moon within the past 100 million years. Nat Geosci 7:787-791
Campbell IH, O’Neill HSC (2012) Evidence against a chondritic Earth. Nature 483:533-558

Canup R (2012) Forming a Moon with an Earth-like composition via a giant impact. Science 338:1052-1055

Cuk M, Stewart ST (2012) Making the Moon from a fast spinning Earth. Science 338:1047-1052

Darwin G (1879) On the precession of a viscous spheroid and on the remote history of the Earth. Philos Trans R Soc Lond 170:447-538

Day JMD et al (2007) Highly siderophile element constraints on accretion and differentiation of the Earth-Moon system. Science $315: 217-219$

Day JMD et al (2010) Osmium isotope and highly siderophile systematics of the lunar crust. EPSL 289:595-605

Demidova SI et al (2014) U-Pb dating of the lunar meteorite Dhofar 1442. Petrology 22:1-16

Elkins-Tanton LT, Grove TL (2011) Water (hydrogen) in the lunar mantle: results from petrology and magma ocean modelling. EPSL 307:173-179

Elkins-Tanton L et al (2002) Re-examination of the lunar magma ocean cumulate overturn hypothesis: melting or mixing is required. EPSL 196:239-249

Gaffney AM, Borg LE (2014) A young solidification age for the lunar magma ocean. Geochim Cosmochim Acta 140:224-240

Giguere TA et al (2000) The titanium contents of lunar basalts. MAPS 25:193-200

Gomes R et al (2005) Origin of the cataclysmic late heavy bombardment period of the terrestrial planets. Nature 435:66-469

Gross J et al (2014) Lunar feldspathic meteorites: constraints on the geology of the lunar highlands, and the origin of the lunar crust. EPSL 388:318-328

Hauri E et al (2015) Water in the Moon's interior: truth and consequences. EPSL 409:252-264

Heldmann $\mathbf{J}$ et al (2015) Evolution of the dust and ice plume components observed by the $\mathrm{L}$ cross mission. Icarus 254:262-275

Hood, L. L. and Jones, J. H. (1987) Geophysical constraints on lunar bulk composition and structure: a reassessment. Proc Lunar Sci Conf 17 in JGR 92 E296-E410

Humayun M, Clayton RN (1995) Potassium isotopic cosmochemistry: genetic implications of volatile element depletion. GCA 59:2131-2148

Jolliff BL et al (2000) Major lunar crustal terranes: surface expressions and crust-mantle origins. JGR (planets) 105:4197-4216

Khan A et al (2006) Are the Earth and Moon compositionally alike? JGR (Planets) 111:1991-2201

Khan A et al (2014) Geophysical evidence for melt in the deep lunar interior and implications for lunar evolution. JGR (Planets) 119:2197-2221

Koeberl C (1986) Geochemistry of tektites and impact glasses. Ann Rev Earth Planet Sci 14:323-350

Koeberl C, Beran A (1988) Water content of tektites and impact glasses and related studies. Proc LPSC 18:403-408

Kohl IE et al (2015) Earth and Moon are indistinguishable in $\partial^{17} \mathrm{O}$ to several parts per million. LPSC Abstr 46:2867

Lodders K (2003) Solar system abundances and condensation temperatures of the elements. Astrophys J 591:1220-1224

Lugmair G, Shukolyukov A (1998) Early solar system timescales according to ${ }^{53} \mathrm{Mn}-{ }^{53} \mathrm{Cr}$ systematics. GCA 62:2863-2886

Magna $\mathrm{T}$ et al (2006) New constraints on the lithium isotopic composition of the Moon and terrestrial planets. EPSL 243:336-353

Mason B, Taylor SR (1982) Inclusions in the Allende meteorite. Smithson Contrib Earth Sci 25:1-30 
Melosh HJ et al (2014) The Moon's upper mantle mostly OPX not olivine. Met Soc Annu Meet 45 abstract 2505

Miljkovic K et al (2015) Excavation of the lunar mantle by basinforming impacts on the Moon. EPSL 409:243-251

Morbidelli A, Levison H (2003) Planetary science: kuiper-belt interlopers. Nature 422:31

Morbidelli A et al (2012) Building terrestrial planets. Ann Rev Earth Planet Sci 409:251-275

Moynier F et al (2006) Isotopic composition of zinc, copper and iron in lunar samples. GCA 70:6103-6117

Moynier F et al (2009) Isotopic fractionation of $\mathrm{Zn}$ in tektites. Earth Planet Sci Lett 277:482-489

Moynier F et al (2010a) Sr stable isotope composition of Earth, the Moon, Mars, Vesta and meteorites. EPSL 300:359-366

Moynier $\mathrm{F}$ et al (2010b) Isotopic fractionation of $\mathrm{Cu}$ in tektites. GCA 74:799-807

Nagaoka $\mathrm{H}$ et al (2014) Implications for the origins of pure anorthosites found in the feldspathic lunar meteorites, Dhofar 489 group. Earth, Planets Space 66:115

Norman MD (2009) The lunar cataclysm: myth or misconception? Elements 5:23-28

Norman MD, Nemchin AA (2014) A 4.2 billion year old impact basin on the Moon: $\mathrm{U}-\mathrm{Pb}$ dating of zirconolite and apatite in lunar melt rock 67955. EPSL 388:387-398

Nyquist L (1977) Lunar Rb-Sr geochronology. Phys Chem Earth 10:103-142

Pahlevan K (2013) Developing the rare earth element constraint for scenarios of lunar origin. LPSC Abs 3073

Pahlevan K, Stevenson DJ (2007) Equilibration in the aftermath of the lunar-forming giant impact. EPSL 262:438-449

Pahlevan K et al (2011) Chemical fractionation in the silicate vapor atmosphere of the Earth. EPSL 301:433-443

Palme H, Jones A (2005) Solar system abundances of the elements. In: Davis AM et al (eds) Treatise on geochemistry, vol 1. Elsevier, Oxford, pp 41-62

Paniello RC et al (2012) Zinc isotopic evidence fir the origin of the Moon. Nature 490:376-379

Petaev MI et al (2014) Testing models of the Moon's origin: condensation of impact-vaporized bulk silicate Earth material. LPSC Abstr 2318

Pritchard ME, Stevenson DJ (2000) Thermal aspects of a lunar origin by giant impact. In: Canup RM et al (eds) Origin of the Earth and Moon. Arizona University Press, Tucson, pp 179-196

Raevskiy SN (2015) On reconciling models of the interior structure of the Moon with gravity data. Phys Solid Earth 51:134-142

Rai $\mathrm{N}$ et al (2014) Lunar core formation: new constraints from metalsilicate partitioning of siderophile elements. EPSL 388:343-352

Reed GW, Jovanovic S (1973) Fluorine in lunar samples. Geochim Cosmochim Acta 37:1457-1462

Robinson KL, Taylor GJ (2014) Heterogeneous distribution of water in the Moon. Nat Geosci 7:401-408

Sedaghatpour F et al (2013) Magnesium isotopic composition of the Moon. GCA 120:1-16

Sharp ZD et al (2010) The chlorine isotope composition of the Moon and implications for an anhydrous mantle. Science 329:1050-1053
Shearer CK et al (2015) Origin of the lunar highlands Mg-suite. Am Mineral 100:294-325

Simon JI et al (2009) Calcium isotopic composition of meteorites, Earth and Mars. Astrophys J 702:707-715

Smith DE et al (2010) Initial observations from the lunar orbiter laser altimeter (LOLA). Geohys Res Lett 37(L18204):1-6

Solomon SC, Longhi J (1977) Magma oceanography I: thermal evolution. Proc LPSC 8:583-599

Stevenson DJ, Halliday AN (2014) Origin of the Moon. Philos Trans A R Soc Lond 372:167-177

Sun SS (1982) Chemical composition and origin of the Earth's primitive mantle. GCA 46:179-192

Taylor SR (1012) Destiny or chance revisited. Cambridge University Press, Cambridge, p 291

Taylor SR (1973) Chemical evidence for lunar melting and differentiation. Nature 245:203-205

Taylor SR (1975) Lunar science: a post apollo view. Pergamon Press, Pergamon $\mathrm{p} 372$

Taylor SR (1982) Planetary science: a lunar perspective. LPI, Houston

Taylor SR (1987) The unique lunar composition and its bearing on the origin of the Moon. GCA 51:1297-1309

Taylor SR (1990) The single impact hypothesis of lunar origin: temperature constraints from lunar elemental abundances. Meteoritics 25:413-414

Taylor SR (2014) The Moon re-examined. GCA 141:670-676

Taylor SR, Jakes P (1975) The geochemical evolution of the Moon. Proc Lunar Sci Conf 5:1287-1305

Taylor SR, Koeberl C (2013) The origin of the Moon revisited. LPSC Abstr 1165

Taylor SR, McLennan SM (2009) Planetary crusts. Cambridge University Press, Cambridge $\mathbf{3 7 8}$ pp

Taylor GJ, Wieczorek MA (2014) Lunar bulk chemical composition. Phil Trans R Soc A 372:20130242

Taylor SR, Taylor GJ, Taylor LA (2006) The Moon: a Taylor perspective. GCA 70:5903-5918

Tera $\mathrm{F}$ et al (1974) Isotopic evidence for a terminal lunar cataclysm. EPS1 22:1-21

Valley JW et al (2014) Correlated $\partial^{18} \mathrm{O}$ and Ti in lunar zircons: a terrestrial perspective for magma temperatures and water content on the Moon. Contrib Mineral Petrol 167:956

Walker D (1983) Lunar and terrestrial crust formation. Proc LPSC 14:B17-B25

Weichert U et al (2001) Oxygen isotopes and the Moon-forming event. Science 294:345-348

Weiss BP, Tikoo SM (2014) The lunar dynamo. Science 346:1198.

Wieczorek MA (2013) The crust of the Moon as seen by GRAIL. Science 339:671-675

Wilhelms DE (1987) The geologic history of the Moon. USGS Prof Paper. p. 1348

Wolf AV, Anders E (1980) Moon and earth-compositional differences inferred from siderophiles, volatiles, and alkalis in basalts. GCA 44:2111-2124

Zhang $\mathbf{J}$ et al (2012) The proto-Earth as a significant source of lunar material. Nat Geosci 5:251-255 